\title{
El abuso de alcohol de los jóvenes en España
}

\author{
Amador Calafat Far \\ Director Adicciones \\ Enviar correspondencia a: \\ Amador Calafat. Rambla, 15, 2 $3^{\text {a }} 07003$ Palma de Mallorca. adicciones@socidrogalcohol.org
}

\section{RESUMEN}

Si bien el consumo global de alcohol está descendiendo en España, estamos asistiendo desde hace años a un incremento del consumo de alcohol por parte de los jóvenes en forma de borracheras o de consumos de alcohol concentrados en pocas horas para alcanzar un cierto nivel de embriaguez, lo que los anglosajones llaman 'binge drinking'. La expresión más típica - aunque no única- de este fenómeno es el botellón, que en realidad no es más que una de las expresiones del consumo de alcohol a lo largo de los espacios recreativos nocturnos del fin de semana.

Preocupa que junto con la posición de liderazgo en el consumo de drogas ilegales en Europa, España esté además incorporando tan rápidamente este patrón de consumo de alcohol que no formaba parte de nuestras tradiciones. Sin embargo no parece que para la sociedad española estos asuntos sean una prioridad tal como lo confirman los estudios sociológicos o la forma en que se ha producido la retirada reciente de la ley que iba a regular el consumo de alcohol por los menores.

Diversas cuestiones se deben tener en cuenta si queremos que la situación actual revierta: el papel activo de las industrias alcoholera y recreativa, el papel globalmente pasivo de los profesionales del alcohol y las drogas en el debate público, la falta de determinación de las autoridades, el discurso mediático, la dificultad que tiene el mundo adulto en posicionarse sobre estas conductas de riesgo,... No comenzar a actuar ahora, hará que todo sea más difícil más adelante.

Palabras clave: jóvenes, alcohol, botellón, binge drinking, politicas sobre el alcohol.

\section{ABSTRACT}

Although the overall consumption of alcohol is falling in Spain we have, for years, been seeing an increase in alcohol consumption by the young. This has taken the form of episodes of drunkenness or quantities of alcohol being drunk within a few concentrated hours to reach a certain level of drunkenness, what the Anglo-Saxons call "binge drinking". The most typical name for this phenomenon -although not the only one- is the botellón which is, in fact, no more than one of the expressions to describe drinking alcohol in the nighttime recreational spaces at the weekend.

It is of some concern that, in conjunction with a leading position in the use of illegal drugs, Spain is also so rapidly incorporating this pattern of alcohol consumption, and one that has never formed part of our traditions. However, it does not appear that these issues are a priority for Spanish society, as has been confirmed by sociological surveys, and by the way in which the law intended to regulate alcohol consumption by minors was recently withdrawn.

Diverse aspects must be taken into account if we wish to see a reversal in the current situation: the active role of the alcohol and recreation industries, the overall passive role of the professionals in alcohol and drugs in public debate, lack of determination by the authorities, the discourse in the media, the difficulty that the adult world has of taking up a position on these behaviours. Failure in not starting to act now will make it all more difficult in the future.

Key words: young people, alcohol, "botellón", binge drinking, alcohol policies.
$\mathbf{L}$ os datos disponibles sobre el consumo de drogas en España entre los jóvenes -y no tan jóvenes- indican que está creciendo. Además, ello nos sitúa como el país con más consumo en Europa en el caso del cannabis o la cocaína, o entre los dos o tres con más consumo para otras drogas'. Pero, muy curiosamente -por denominarlo de alguna forma-, la realidad es que la preocupación por el tema de las drogas entre los españoles está siguiendo exacta- mente la dirección opuesta. En el último estudio del Centro de Investigaciones Sociológicas ${ }^{2}$ de febrero de este año sólo un 1,2\% de los españoles mayores de 18 años creen que la droga actualmente es el principal problema de España. Esto significa que hay otras preocupaciones prioritarias, y ésta, en concreto, sólo se sitúa entre el $10^{\circ}$ y $12^{\circ}$ lugar, empatado con otros dos temas. Sin embargo en marzo de 1997 el tema de las drogas ocupaba el tercer lugar en importancia 
tras el paro y el terrorismo ${ }^{3}$. Es evidente que preocuparse por una cosa no es en si mismo una garantía de que un problema se solucione. Pero no menos cierto es que sin una mínima preocupación va a ser más difícil poner los medios para buscar, encontrar y exigir soluciones. Hay diversas lecturas posibles acerca de este interés decreciente por el tema de las drogas. Podemos pensar que nuestra sociedad sólo reacciona ante problemas planteados de forma dramática en los medios de comunicación $y$, desde que ya no se produce la violencia ligada al uso de heroína, parece como si ya el problema fuese menor. Pero también es posible que la menor preocupación se deba a un cambio de la percepción social de cómo se deben enfocar los problemas, por el cual el consumo de alcohol y otras drogas tiende a verse cada vez más como algo que compete exclusivamente al ámbito del individuo. Habría como una colonización de 'lo público' por 'lo privado'; es decir ahora parece que es el individuo 'aislado' quien decide consumir y, por tanto, es él/ella quien tiene que cuidar acerca de si le va bien o mal. De esta forma, deja de ser una preocupación social ante la cual actuar de forma colectiva. Con ello se desactiva una visión desde la salud pública sobre el tema y, paralelamente, la sociedad en su conjunto deja de actuar. De ahí la importancia de rescatar esta visión de salud pública para el tema de las drogas si pretendemos generar políticas eficaces, que tengan el respaldo de la sociedad. Y ello por muchas razones: porque afecta a terceras personas, porque tiene un coste económico y social para la sociedad, porque hay menores implicados sobre los que hay un deber de tutelaje; pero sobretodo, porque la lógica que lleva al consumo de drogas es una construcción social y cultural.

Y ¿qué pasa con el alcohol? La situación es calcada a la anterior. El problema también va creciendo entre los jóvenes, pero al igual que con las drogas ilegales existe poca conciencia de la responsabilidad social que explica porque esto ocurre -excepto en los aspectos más mediáticos como son el botellón- y, en consecuencia, las autoridades pertinentes no se atreven con algún tipo de soluciones por miedo a la impopularidad. Hay dificultades para actuar y se deja el tema en manos de cada individuo consumidor, al cual se le pide -en un alarde de pretendida comprensión y respeto por el mundo adolescente y juvenil- que sea el mismo el que se regule. Este enfoque es estrictamente coincidente con la visión que mantiene la industria alcoholera, la cual pone el acento en el 'consumo responsable' y en la 'educación y prevención' y está en contra de otras formas de regulación más ambiciosas y complejas por ser acciones de compromiso colectivo y límites éticos del mercado. En la web del Portman Group (que representa a las alcoholeras inglesas y mundiales más importantes) se puede leer textualmente: “Un enfoque basado en la 'educación y la prevención' es más eficaz que medidas de control que afecten a grupos. Las medidas de reducción del daño sobre el alcohol debería estar dirigidas a la minoría que hacen un mal uso del alcohol en lugar de a la mayoría que hace un uso responsable". Precisamente las medidas que apoya la industria como positivas son las que no han demostrado su eficacia o ésta es mínima. Medidas como la educación en las escuelas (que en la práctica supone muchas veces aplicar programas que en su gran mayoría no han sido evaluados o no se aplican correctamente) o el conductor designado, que tanto gusta a la industria, no han demostrado su eficacia o es muy limitada en las condiciones actuales de aplicación. Por el contrario sí han demostrado ser efectivas las medidas de control sobre el acceso (aumentar la edad, impedir el accesos a menores, aumentar los precios,... $)^{8}$. El problema, el gran problema, es que el discurso del individuo 'responsable' ha cautivado al gran público, quien también cree que estas medidas son más eficaces y más populares, con la ventaja añadida que no le hacen entrar en contradicción. No hay que hacer nada, todo lo tiene que hacer el que bebe. Y los políticos, que en teoría están más informados, pues están más cercanos a los técnicos, dudan en poner en marcha medidas que pueden ser impopulares, y muchas veces no actúan hasta que los problemas desbordan y los medios de comunicación exigen soluciones. Pero, para entonces, ya muchas veces ciertos fenómenos como el botellón han crecido tanto que no es fácil revertir la dinámica.

La prueba más fehaciente de la situación en la que nos encontramos en términos públicos ha sido la facilidad y la demagogia con la que se ha podido criticar el Proyecto de Ley de prevención del consumo de alcohol en menores impulsado por el Ministerio de Sanidad y Consumo español. Y la prueba también ha sido la falta de voluntad política en mantener el proyecto ante estas críticas sin fundamento científico, ni basadas en el interés de la mayoría de los jóvenes. Un fenómeno similar se produjo durante la anterior legislatura en relación con lo que entonces se denominó en la calle 'Ley antibotellón', aunque en aquella ocasión se habían cruzado los papeles. Ambas leyes defendían cuestiones muy elementales como controlar el acceso de los menores al alcohol, regular la publicidad que va dirigida a ellos, prohibir el consumo en las vías públicas de los menores de 18 años. Se trataba en ambos proyectos de una cuestión de mínimos, que sólo afectaba al consumo de los menores de edad. Difícilmente había algo que objetar a estas leyes. En algunos casos ya existían leyes más estrictas a nivel autonómico. ¿Dónde ha estado pues el problema para que estas leyes no prosperaran? Claramente en la presión de la industria alcoholera, en la poca conciencia popular hacia el problema, en una opinión pública poco formada y en la utilización partidista de cuestiones que claramente requieren un pacto de estado. En el Reino Unido los profesionales parecen tener la misma sensación: "la influencia de la industria parece 
estar dictando las grandes líneas de la política antialcohol inglesa, consiguiendo que sea a grandes rasgos ineficaz"3.

¿Cuál es la situación real respecto al alcohol entre jóvenes españoles? Sin lugar a dudas no muy optimista. En este caso las frecuentes menciones en los medios de comunicación al fenómeno del botellón no son una casualidad o una situación recreada por los periodistas, como tampoco lo son los accidentes de tráfico de fin de semana o el aumento de la violencia. Es cierto que el consumo de alcohol per capita de toda la población española está descendiendo ${ }^{4}$ al igual que ocurre en la mayoría de países europeos. Desde los consumos máximos registrados de 14,2 litros de alcohol puro de mediados de los 70 en el caso español, hemos pasado a 10 litros en el 2003, lo cual es sin lugar a dudas un importante descenso, aunque menor que los conseguidos por otros países de nuestro entorno como Francia e Italia, que partían de consumos más altos. Ello nos sitúa en la posición número ocho entre los países europeos). Desde los estudios de Lederman ${ }^{5}$ se relaciona el consumo global de una población con el porcentaje de consumidores de riesgo que hay dentro de esta población. "Los que hemos abogado por la reducción del daño, hemos prestado poca atención al hecho de que si queremos realmente reducir los problemas relacionados con el alcohol mucha gente tiene que beber bastante menos. No existe una razón lógica o explicable porque todo el mundo tiene que beber menos y no sólo los que beben en exceso. Pero la forma más efectiva de reducir el nivel global de las tragedias y violencias relacionadas con el alcohol pasa por poner un tapón a nuestro nivel de consumo nacional" según Martín y Moira Plant ${ }^{6}$. Pero también es cierto que el consumo global y los consumos excesivos se distribuyen de distinta manera según los países. En este sentido desde hace tiempo se viene hablando de las diferencias norte y sur, puesto que la práctica de episodios de consumo compulsivo (binge drinking) -o de 'beber concentrado' como propondríamos nosotros denominar a esta forma de consumo- en nuestro país, así como en otros países mediterráneos, es tradicionalmente menor. Pero las cosas están cambiando bastante rápidamente, especialmente entre los jóvenes y menores.

\section{CAMBIOS NORTE-SUR}

Existe la dicotomía convencional norte-sur en Europa en relación con el consumo de alcohol. Desde ese estereotipo en los países mediterráneos viticultores, se bebe más vino, sobre todo en las comidas, y se emborrachan poco. Al contrario, los países del centro y norte de Europa son bebedores de cerveza y des- tilados, no beben en las comidas y se emborrachan con cierta frecuencia, sobre todo los fines de semana. Ese estereotipo ya no se mantiene del todo vigente. Las cosas van cambiando y habría que tomar nota de los cambios sociales que se están produciendo. Por ejemplo, sigue siendo cierto que Italia, Portugal y Francia están entre los que más dicen consumir a la hora de comer, pero ya en Suecia y Dinamarca hay más personas que en España que dicen sólo consumir cuando comen. En el último Eurobarometro ${ }^{7}$ realizado sobre actitudes hacia el alcohol, resulta -muy sorprendentemente- que los españoles son los que con mayor frecuencia (un 28\%, comparado por ejemplo con un $12 \%$ de los ingleses) han consumido varias veces -cinco o más consumiciones- en una sola ocasión durante una semana.

Pero donde los cambios se dan más rápidamente -sobre todo en el sentido de una homogeneizaciónes entre los jóvenes de los países europeos. Aquí si que los viejos estereotipos ya no funcionan igual. Por ejemplo, los varones jóvenes ingleses beben con más frecuencia a lo largo de la semana que los de Francia ${ }^{8}$. También son los jóvenes británicos quienes consumen alcohol en las comidas con la misma frecuencia que los italianos y más que los franceses ${ }^{9}$. En general, la tendencia entre los jóvenes europeos que consumen alcohol es a no beber durante las comidas (especialmente esto ocurre, aunque parezca paradójico, en el sur), a emborracharse los fines de semana y a preferir la cerveza. Y otro aspecto fundamental del cambio es el consumo de las mujeres jóvenes. Se está reduciendo rápidamente en casi todos los países y en algunos países las mujeres ya consumen y abusan de alcohol más que los varones. Es probable que ese aumento del consumo femenino sea un indicador muy alarmante en términos de salud pública, pero también preocupa la dirección que ha tomado en algunas cuestiones la emancipación femenina.

\section{BORRACHERAS O 'BEBER CONCENTRADO' (BINGE DRINKING) EN JÓVENES}

Recientemente ha surgido con fuerza el termino anglosajón "binge drinking" para referirse a la forma preferente de consumo de alcohol actual de muchos adolescentes, aunque ya los adultos, sobre todo de países anglosajones, lo vienen practicando tradicionalmente. Incluso entre los profesionales españoles ha prendido esta denominación, seguramente porque no tenemos una traducción fácil a nuestro idioma. Aunque muchas veces la consecuencia del 'binge drinking' acaba siendo la borrachera, no son sin embargo la misma cosa. El termino 'binge' en inglés se refiere a esta forma compulsiva de consumo, que en el caso de la comida denominamos atracón. Al igual que 
los esquimales tienen muchas palabras para designar distintos tipos de nieve, algunas culturas tienen un mayor número de vocablos para la borrachera. A este respecto los ingleses, los holandeses y los suecos tienen más palabras que se relacionan con la borrachera que otras culturas ${ }^{10}$. Entre nosotros hemos utilizado en el pasado en alguna ocasión el término beber compulsivo, pero quizás esta palabra tiene connotaciones psiquiátricas que dificultan la adecuación del término. Posiblemente 'beber concentrado' es un termino que está menos contaminado, que es breve y que se refiere a esta característica de beber bastante en poco tiempo.

En noviembre del 2003 hubo una reunión científica en Washington para definir el termino 'binge drinking' convocada por la Nacional Institute on Alcohol Abuse and Alcoholism (NIAAA). La definición que se consensuó entonces sería el de consumir 5 o más bebidas para el varón y 4 o más para la mujer dentro del espacio de unas dos horas. Pero existen otras definiciones al uso todavía como pueden ser beber hasta alcanzar o superar la tasa de alcoholemia de 0,08. Sea como sea el hecho mediático ha llevado a que el término haya hecho fortuna incluso en los ambientes académicos.

Con una u otra definición el hecho real es que los jóvenes europeos se están emborrachando cada vez más. En un estudio ${ }^{11}$ que se realiza cada 4 años entre bastantes países europeos desde 1995 -el último abarca 35 países o territorios, aunque no incluye España- y dirigido a estudiantes de 15 a 16 años denominado ESPAD, se les pregunta si han bebido por lo menos cinco o más consumiciones en una sola vez por lo menos tres veces durante el último mes. En la mayoría de los países ha crecido esta práctica.

En relación a España tenemos datos sobre el "beber concentrado" a través de SIVFRENT (Sistema de vigilancia de factores de riesgo de enfermedades no transmisibles), que es una serie de estudios epidemiológicos que se viene realizando en la comunidad de Madrid desde el año 1996 hasta la actualidad y que recoge tanto este tema como muchos otros ${ }^{12}$. En concreto la frecuencia de 'beber concentrado' (en este caso concreto se define como el consumo de $60 \mathrm{gr}$ de alcohol puro en una sesión durante los últimos 30 días) entre población escolar de 15 a 16 años -lo cual nos permite una comparación con el estudio europeo citado antes ESPAD- es del 35,5\% (38,5\% en los varones y $32,1 \%$ en las mujeres) en el año 2005. Estas frecuencias nos sitúan entre los países de mayor frecuencia dentro del panorama europeo. El dato positivo sería que por lo menos las frecuencias alcanzadas siguen más bien en dicha comunidad una tendencia a la baja desde el pico de $53,2 \%$ para los varones alcanzado en 1997.
Otro aspecto que nos parece muy importante es que cada vez más empiezan a pesar a la hora de decidir beber concentradamente las expectativas positivas que se tienen hacia este tipo de consumo que las negativas, sobre todo en el caso de los jóvenes ${ }^{13,14}$. Similares conclusiones las encontramos en diversos estudios como el ESPAD ${ }^{11}$, las encuestas escolares del PND o, refiriéndose a otras drogas, en otros estu$\operatorname{dios}^{15,16}$. Esto es de una trascendencia enorme cara a la prevención. Ya no va a ser suficiente con aumentar la percepción de riesgo, que ha sido la forma clásica de abordar el tema. Sino que habrá que conseguir tener en cuenta 'la percepción de las ventajas' para que beber en exceso sea algo culturalmente no apetecible, y esto supone una forma muy distinta de hacer prevención.

Además, debemos recordar que las expectativas sobre el alcohol se forman ya años antes de que se empiece a consumir ${ }^{17}$. Hay estudios que muestran que a los 8 años ya se tiene una idea sobre la conveniencia de tomar alcohol ${ }^{18}$. Y, normalmente, a medida que avanza la edad, estas expectativas se van haciendo cada vez más positivas. Además estas expectativas medidas en la adolescencia temprana sirven para predecir no sólo la iniciación en el consumo, su intensidad y frecuencia, sino que también predicen el uso problemático al final de la adolescencia y al inicio de la juventud ${ }^{19}$.

\section{LA CUESTIÓN DEL GÉNERO}

También la situación actual presenta nuevos retos en cuestiones que hace no tanto tiempo parecían inamovibles. Siempre habíamos visto que el consumo de alcohol y otras drogas, junto con la violencia y conductas similares, era una cuestión sobre todo ligada a lo masculino, mientras que el malestar femenino se expresaba más como depresión ${ }^{20}$. Posteriormente hemos presenciado como las frecuencias de consumo se han ido acercando y, en el caso del tabaco, ya hace tiempo que entre los jóvenes de diversos países ya es más una cuestión femenina que masculina. Pero los abusos, incluidos los referidos al alcohol, seguíamos pensando que eran más cosa de los hombres. Pero en el último ESPAD ${ }^{11}$, entre jóvenes europeos escolarizados de 15 a 16 años resulta que en tres países (Irlanda, Noruega y Reino Unido) las escolares superan a sus compañeros varones en la práctica del beber concentrado (binge drinking). Es interesante además comprobar cómo la visión tradicional norte sur es la que funciona menos cuando nos referimos sobre todo a mujeres jóvenes. De esta forma Reino Unido e Italia muestran consumos similares en cuanto a chicas jóvenes (por la franja alta), mientras que Finlandia y Francia también son comparables pero en la franja baja9 . 
En un estudio cualitativo utilizando entrevistas en profundidad $^{21}$ a 64 adolescentes ingleses de 14 a 17 años de ambos sexos, indicaron frecuencias parecidas de incidentes negativos relacionados con el 'beber concentrado', no obstante las mujeres se quejaban más de experiencias sexuales no deseadas y de encontrarse mal físicamente, mientras que los varones se quejaban de peleas.

\section{EL BOTELLÓN}

El botellón es una expresión paradigmática en el caso español de esta forma de beber concentrado ${ }^{22}$. En toda Europa los jóvenes tienden a beber en lugares públicos, principalmente bares o discotecas, a veces también en las calles o plazas y, sobre todo, cerca de los bares o pubs. Pero no cabe duda que en el caso español a través del botellón se han superado todas las expectativas. Lo que más debería preocupar desde la perspectiva de salud pública son los aspectos culturales positivos que los jóvenes asocian a este fenómeno, que lo viven como una creación expresamente suya, y que una parte de los adultos también de alguna forma apoyan o justifican. El discurso romántico optimista que justifica las acciones de los jóvenes como formas de trasgresión, o de identidad propia hace un flaco favor a los jóvenes, pues casi nunca es verdad que detrás de estas manifestaciones de los jóvenes haya un esfuerzo hacia la emancipación y la autonomía personal. Ese discurso contribuye, sin querer muchas veces, a disfrazar la cada vez mayor inmersión de los jóvenes en una economía consumista que les seduce, los atrapa y los orienta hacia estilos de vida, cuyas consecuencias últimas serán mayores desigualdades y más frustraciones. En definitiva no es tan claro que la defensa violenta que han hecho algún grupo de jóvenes de la necesidad de hacer botellón, suponga un acto de emancipación contra los adultos, sino más bien el deseo de seguir disfrutando de un ocio consumista sin obligaciones y sin imposiciones de parte de los adultos. Más allá de ser un recurso cultural para los jóvenes donde la clave es consumir alcohol, lo que también preocupa es como el botellón desactiva las medidas preventivas que se han demostrado más eficaces en el control del consumo excesivo, como pueden ser aumentar los precios, disminuir la accesibilidad y controlar el acceso de los menores.

El botellón es un fenómeno social complejo que responde a distintas causas y cumple distintas funciones (socializadoras, reivindicativas, etc). Pero parecería que el consumo de alcohol cumple una función realmente clave ${ }^{22}$ en todo el entramado. Para bastantes de los asistentes se trata de beber todo lo que se pueda por el dinero que se tiene. Un fenómeno similar ocurre en el Reino Unido donde ha bajado el consumo en los pubs, pero ha subido el consumo en casa de los adolescentes, puesto que no pueden beber fácilmente en la calle. Se llama 'pre-drinking' a esta forma de beber que empieza en casa antes de salir por las noches pues es más barato hacerlo de esta forma.

\section{CONCLUSIONES}

El problema del consumo abusivo entre los jóvenes españoles está creciendo desde hace años, en lo que representa una nueva forma de beber más compulsiva que podemos denominar 'beber concentrado' (termino por el que se puede traducir el 'binge drinking' inglés). Esta forma de beber es nueva entre nosotros y, hasta ahora, ha sido muy poco frecuente entre los adultos. Sin embargo, hay que prever que en un futuro inmediato no sólo será típica entre los adolescentes y jóvenes, sino que a medida que vayan creciendo conservarán esta nueva forma de beber en su paso a la adultez, que antes solo atribuíamos a los países centro europeos o anglosajones. Además, esta nueva forma de beber se asocia a más problemas como son: afectación de la maduración cerebral en los adolescentes, mayor capacidad de generar problemas con el alcohol, problemas con la conducción, violencia, prácticas de riesgo sexuales, etc.

Esta evolución responde a cambios culturales profundos. Las transformaciones culturales en el ámbito de los jóvenes se producen muy rápidamente en el ámbito internacional y suponen una gran homogeneización de las pautas de consumo en Europa en esta franja de edad. Estos cambios se incluyen dentro de la incorporación masiva de los jóvenes a los hábitos recreativos de fin de semana, en los que España es pionera. Dichos hábitos no son precisamente una forma de resistencia o de construcción de la identidad juvenil autónoma, sino más bien el fruto de la presión de una poderosa presión económica y cultural que conduce a muchos jóvenes a estilos de vida evasivos o fragmentados por la compulsión. Detrás de todo ello están las alcoholeras, pero mucho más importante es la industria de la diversión, con un poder clave no sólo en el orden económico o de influencias políticas, sino en crear imaginario colectivo, en pensar que es lo que puede gustar a los adolescentes y jóvenes para que consuman más.

Sin embargo, la sociedad española no parece en exceso alarmada por estos nuevos consumos, ni por estos cambios culturales. Hay algunas excepciones a la regla, como cuando se generan problemas de convivencia vecinal, o bien, temporalmente, cuando se habla de ello en los medios de comunicación, pero enfocado sólo como un problema entre colectivos (los que se divierten y los que quieren descansar). Tampo- 
co parece que haya mucha preocupación por habernos convertido en el primer, o uno de los principales países europeos en cuanto al consumo de drogas ilegales. No se han encendido las alarmas y tampoco parece claro que se vayan a encender si no se hace una labor por parte de todos y cada uno de los profesionales y de las organizaciones que les representan. Los fracasos recientes en esta y en la anterior legislatura en la tramitación de leyes que regulen y protejan el consumo de alcohol por los menores de edad pueden tener un alcance mayor del que parece. Cabe pensar que la tentación y lo fácil para los dos partidos mayoritarios va a ser pasar de puntillas sobre este problema pues los dos han quedado marcados por su postura. ¿Habrá que esperar pues hasta que la situación crezca a unos niveles insoportables que llegue a colmar la opinión pública? Si esta es la alternativa ello puede ser terrible, pues puede pasar bastante tiempo antes de que la sociedad reaccione. Porque así como se ha llegado a la 'normalización' del consumo de drogas, con los mismos criterios se puede llegar a la 'normalización' de las consecuencias del consumo de alcohol y otras drogas (violencia, sexualidad de riesgo, anomia, malestar, desigualdades,...).

Como apunte final, puede ser interesante reflexionar sobre los datos comparados que nos ofrece el último eurobarométro específico sobre temas de alcohol ${ }^{7}$. Se plantean allí dos preguntas y los encuestados deben elegir cual se acerca más a lo que piensan. ¿Son los individuos suficientemente responsables para protegerse ellos mismos de los problemas relacionados con el alcohol?, o bien, ¿deben ser las administraciones públicas las que tienen que intervenir para proteger a los individuos? Los españoles eligen mayoritariamente $(55 \%)$ la opción de que es el estado quien debe actuar para proteger al individuo y sólo nos superan en esta visión Hungría (un 57\%) e Italia (un 56\%). Como es lógico también estamos entre los que menos creen (un 39\%) que sea el individuo el responsable de cuidarse a si mismo, solo superados en esta opción por los dos mismos países. Visto lo visto nos encontramos con la irónica paradoja que países que apoyan generalmente una visión individualista como España e Italia, son luego los países que confían más en el Estado para solucionar los problemas, mientras que en los países donde las cuestiones de salud pública se toman más en serio (países escandinavos, Reino Unido, Alemania, Francia,...) esperan más de la responsabilidad del individuo. Existe cada vez más un debate que diferencia lo que es el individuo y lo que es el ciudadano ${ }^{23}$, que quizás puede ayudar a explicar esta aparente paradoja. En nuestro país nos decantamos por ser individuos sin responsabilidad ciudadana. Es la 'sociedad' -un ente abstractola que debe preocuparse de los problemas públicos, $y$, si puede ser, debe hacerlo de forma que moleste poco. Pero, eso si, requerimos que la sanidad públi- ca esté allí presta a resolver nuestros problemas si la necesitamos. Creemos que el ideal es vivir sin prohibiciones, sin imposiciones sociales, pero acudiendo al sistema público cuando nuestra conducta nos trae problemas. Volviendo a los resultados de la encuesta referidos a España, entendemos que lo que ocurre es que los individuos ceden su responsabilidad a la sociedad para que les soluciones los problemas. Pero posiblemente el buen funcionamiento de los servicios públicos está en relación con una mayor responsabilidad ciudadana. ¿Será esta la lección a aprender? En fin, tarea nos queda.

\section{REFERENCIAS}

1 European Monitoring Centre for Drugs and Drug Addiction (EMCDDA) The state of the drugs problem in Europe. Lisboa: European Monitoring Centre for Drugs and Drug Addiction; 2006.

2 Centro de Investigaciones Sociológicas. Barómetro febrero $2007 n^{\circ}$ 2677. 2007. Disponible en http://www. cis.es/cis/opencms/-Archivos/Marginales/2660_ 2679/2677/e267700.html [consultado el 20/05/07]

3 Centro de Investigaciones Sociológicas. Barómetro febrero 1997; n²241. 1997. Disponible en dhttp:// wWw.cis.es/cis/opencms/ES/1_encuestas/estudios/ ver.jsp?estudio=1231 [consultado el 20/05/07].

4 World Drink Trends 2005. A Carefully Distilled Collection of Global Drinks Data. Oxfordshire: World Advertising Research Center, 2007.

5 World Health Organization Regional Office for Europe. European health for all database (HFA-DB).Updated: June 2006. Disponible en: http://data.euro.who.int/ hfadb/ [consultado el 10/04/07]

5 Ledermann S. Alcool, alcoolism, alcoolisation. Paris: Presses Universitaires de France, 1956.

6 Plant M, Plant M. Binge Britain. Alcohol and the National Response. New York: Oxford University Press, 2006.

7 TNS Opinion \& Social. Special Eurobarometer 272b / Wave 66.21. Attitudes towards alcohol. 2007. Disponible en http://ec.europa.eu/public_opinion/ archives/ebs/ebs_272b_en.pdf [consultado el 10/05/07]

8 Anderson P, Baumberg B. Alcohol in Europe. A public health perspective. London: Institute of Alcohol Studies, 2006.

9 Leifman H. A Comparative Analysis of Drinking Patterns in 6 EU Countries in the Year 2000. Contemporary Drugs Problems 2002; 29:501-548.

10 Cameron D, Thomas M, Madden S, Thornton C, Bergmark A, Garretsen H et al. Intoxicated accross Europe: In search of meaning. Addiction Research 2007; 8(233):242. 
11 Hibell B, Andersson B, Bjarnason T, Ahlström S, Balakireva O, Kokkevi A et al. The ESPAD Report 2003: alcohol and other drug use among students in 35 European countries. Stockholm: The Swedish Council for Information on Alcohol and Other Drugs (CAN) and The Pompidou Group at the Council of Europe, 2004.

12 Instituto de Salud Pública. Hábitos de salud en la población juvenil de la Comunidad de Madrid 2005. Boletín epidemiológico de la Comunidad de Madrid 2005; 11:3-42.

13 Leigh BC, Stacy AW. Alcohol expectancies and drinking in different age groups. Addiction 2004; 99(2):215-227.

14 Aas HN, Leigh BC, Anderssen N, Jacobsen R. Twoyear longitudinal study of alcohol expectancies and drinking among Norwegian adolescents. Addiction 1998; 93(3):373-384.

15 Calafat, A, Stocco, P, Mendes, F, Simon, J, Van Wijngaart, G, Sureda, P, et al (1998) Characteristics and Social Representation of Ecstasy in Europe. Palma de Mallorca. IREFREA.

16 Martino, SC, Collins, RL, Ellickson, PL, Schell TL, McCaffrey, D. Socio-environmental influences on adolescents' alcohol outcome expectancoes analysis. Addiction 2006; 101:971-983.
17 Johnson HL, Johnson PB. Children's alcohol-related cognitions: positive versus negative alcohol effects. J Alcohol Drug Educ 1995; 40:1-12.

18 Casswell S, Gilmore LL, Sliva P, Brasch P. What children know about alcohol and how they know it. Addiction 1988; 83:223-227.

19 Christiansen BA, Smith GT, Rochling PV, Goldman M. Using alcohol expectancies to predict adolescent drinking behavior after one year. J Consult Clin Psychol 1989; 57:93-99.

20 Calafat A, Amengual M, Palmer A, Saliba C. Drug Use and Its Relationship to Other Behavior Disorders and Maladjustment Signs among Adolescents. Subst Use Misuse 1997; 32:1-24.

21 Coleman L, Cater S. Underage 'binge' drinking: a qualitative study into motivations and outcomes. Drugs: education, prevention and policy 2005; 12(2):125-136.

22 Calafat A, Juan M, Becoña E, Castillo A, Fernández C, Franco $\mathrm{M}$ et al. El consumo de alcohol en la lógica del botellón. Adicciones 2005; 17(3):193-202.

23 Bauman Z Modernidad líquida. Méjico: Fondo de Cultura Económica. 2004. 
